participants from across the UK were asked to keep a diary of everything they ate and drank over four days. Diaries were coded and intake frequencies of foods derived using groups of nutritionally similar foods. For the $2 \%$ of participants who had three-day diaries, the frequencies were multiplied by $4 / 3$. Principal component analysis (PCA) was performed on frequencies of consumption. Reduced-item prudent diet scores were calculated by multiplying coefficients for the ten most characteristic foods by each individual's standardized reported frequency of consumption. Prudent diet scores calculated for both the full and 10-item prudent diet scores were standardised. Willing participants provided a blood sample; vitamin C status was measured on the BMG Labtech FLUOstar OPTIMA plate reader and beta carotene status by high performance liquid chromatography.

Results 6090 adults (aged 20 to 96 years) provided dietary data in the first eight years of the NDNS (2008-2016). PCA of intake frequencies of 126 food groups revealed a prudent diet pattern characterised by high intakes of wholemeal bread, vegetables, fruit, water, oily fish, yoghurt and high-fibre breakfast cereals, and low intakes of white bread, sugar, sugarsweetened soft drinks and chips. Pattern coefficients were very similar when analyses were conducted separately in males and females, and in those aged less than 65 and $65+$ years. The 10 -item prudent diet score was calculated based on foods with the five highest and five lowest coefficients. Spearman's correlation between the full and 10-item prudent diet scores was 0.84 . The mean difference between the full and 10-item prudent diet scores was 0.00 SDs with Bland-Altman limits of agreement -1.89 to $1.89 \mathrm{SD}$. Notable correlations were seen between the full prudent diet score and vitamin $C\left(r_{s}=0.43\right)$ and beta-carotene status $\left(r_{s}=0.45\right)$; these were only slightly attenuated for the 10 -item prudent diet score $\left(r_{s}=0.40\right.$, $\mathrm{r}_{\mathrm{s}}=0.38$ respectively).

Conclusion A 10-item prudent diet score based on foods that characterise the prudent dietary pattern describes a healthy diet. A 10-item FFQ would have clear advantages for time and resources, and may provide an appropriate tool to describe UK diets while reducing participant burden.

\section{OP108 A PROSPECTIVE COHORT STUDY ON NUT AND PEANUT BUTTER INTAKE AND THE RISK OF ENDOMETRIAL AND OVARIAN CANCER}

'L Nieuwenhuis*, 1,2PA van den Brandt. 'Epidemiology, Care and Public Health Research Institute (CAPHRI), Maastricht University, Maastricht, Netherlands; ${ }^{2}$ Epidemiology, GROW School for Oncology and Developmental Biology, Maastricht University, Maastricht, Netherlands

\subsection{6/jech-2019-SSMabstracts.40}

Background Nut intake has been associated with reduced cancer-related mortality and cancer risk. However, very few studies investigated the association between nut consumption and the risk of endometrial and ovarian cancer, with inconclusive results. We prospectively examined the relation between total nut, tree nut, peanut, and peanut butter intake and the risk of endometrial and ovarian cancer in the prospective Netherlands Cohort Study (NLCS).

Methods In 1986, 62,573 women aged 55-69 years were included in the NLCS. At baseline, all participants filled in a questionnaire and a subcohort of 2,589 women was randomly selected. After 20.3 years of follow-up, 389 endometrial and 347 ovarian cancer cases with complete data were included in the analysis. Hazard ratios (HRs) were calculated in multivariable-adjusted Cox regression analyses, using a case-cohort approach.

Results Compared to nonconsumers, the HRs (95\% confidence intervals) for women consuming $10+\mathrm{g}$ total nuts/day were $1.23(0.82-1.87)$ for endometrial cancer and $0.84(0.57-1.24)$ for ovarian cancer. For tree nut, peanut, and peanut butter intake, also no significant relations with endometrial or ovarian cancer were observed. In the endometrial cancer analyses, significant interactions of total nut intake with body mass index and cigarette smoking status were found.

Conclusion The results of this study suggest that intake of total nuts, tree nuts, peanuts, and peanut butter is not related to the risk of endometrial or ovarian cancer. The observed interactions in the endometrial cancer analyses, particularly by cigarette smoking status, require confirmation in other studies.

\section{Health Inequalities 1}

\section{OP40 THE ASSOCIATION BETWEEN CHILDHOOD SOCIO- ECONOMIC POSITION AND ADVERSE CHILDHOOD EXPERIENCES (ACES): A SYSTEMATIC REVIEW}

${ }^{1} \mathrm{D}$ Walsh ${ }^{*},{ }^{2} \mathrm{G}$ McCartney, ${ }^{3} \mathrm{M}$ Smith, ${ }^{4} \mathrm{G}$ Armour. ${ }^{1} \mathrm{GCPH}$, Glasgow Centre for Population Health, Glasgow, UK; ${ }^{2}$ Public Health Observatory, NHS Health Scotland, Glasgow, UK; ${ }^{3}$ Mental Health Services, NHS Greater Glasgow and Clyde, Glasgow, UK; ${ }^{4}$ Library and Knowledge Services, NHS Health Scotland, Glasgow, UK

\subsection{6/jech-2019-SSMabstracts.41}

Background An increasing amount of evidence has emerged in recent years of the association between different aspects of childhood adversity (termed ACEs - Adverse Childhood Experiences - in a great many studies) and increased risks of negative outcomes in later life. Consequently, ACEs have become a policy priority in many quarters. Despite most ACEs being highly socially patterned, there has been very little discussion in the political discourse regarding the role of childhood socio-economic conditions in understanding and addressing them. The aim, therefore, was to investigate what is known about the association between childhood socio-economic position (SEP) and ACEs, including the extent to which the former explains the latter.

Methods Relevant databases (MEDLINE; psycINFO; ProQuest Public Health Database, Cochrane Library) were searched for all papers satisfying four inclusion criteria: (1) measurement of social position in childhood (prior to ACEs measurement); (2) measurement of multiple aspects of childhood adversity; (3) childhood adversity was the outcome; (4) statistical quantification of the relationship between childhood SEP and childhood adversity. Non-English language papers were excluded.

The initial search terms included ACEs, SEP, and their synonyms. A second search additionally included 'maltreatment'. Papers were independently screened and critically appraised by two authors. Risk of bias was assessed, and overall study quality calculated using a modified version of the Hamilton Tool. Results were synthesised narratively because of the wide variation in definitions of exposures and outcomes.

Results For the ACEs-based search, 2,779 papers were screened, of which 52 were reviewed in full-text. Of these, only six were eligible for qualitative synthesis. The second search (including maltreatment) increased the numbers to: 4,463 papers screened; 166 full texts; 35 included for 
synthesis. 18 papers were deemed to be 'high' quality, five 'medium', the rest 'low'. Meaningful statistical associations were observed between the exposure (childhood SEP) and the outcome (ACEs/maltreatment) in the vast majority of studies, including all bar one of those deemed to be high quality. Low SEP is therefore clearly a determinant of ACEs/maltreatment: the longitudinal nature of many studies means the association is most likely causal.

Conclusion The relationship between childhood SEP and ACEs is clear, but under-researched. More evidence exists in the maltreatment literature. With UK child poverty levels predicted to increase markedly, any policy approach which ignores the socio-economic context to ACEs is flawed. Policy needs to help those currently affected by childhood adversity; but to prevent further adversity, it must also address the key socioeconomic drivers.

\section{OP63 TRAJECTORIES OF SOCIO-ECONOMIC POSITION FROM BIRTH TO ADULT AGE AND SUBSEQUENT MORTALITY: THE UPPSALA BIRTH COHORT MULTIGENERATIONAL STUDY}

${ }^{1,2}$ | Koupil*, ${ }^{1} \mathrm{~A}$ Heshmati, ${ }^{1,3} \mathrm{~A}$ Goodman, ${ }^{4} \mathrm{G}$ Mishra. ${ }^{1}$ Dept. Public Health Sciences, Stockholm University, Stockholm, Sweden; ${ }^{2}$ Dept. Public Health Sciences, Karolinska Institutet, Stockholm, Sweden; ${ }^{3}$ Faculty of Epidemiology and Population Health, London School of Hygiene and Tropical Medicine, London, UK; ${ }^{4}$ School of Public Health, University of Queensland, Brisbane, Australia

\subsection{6/jech-2019-SSMabstracts.42}

Background Several theoretical life course models (critical period, sensitive period, accumulation) have been proposed, all of which may be relevant for understanding of when and how socioeconomic inequalities in health arise. Our aim was to investigate whether the effect of socio-economic position on all-cause mortality accumulates over the life course or if some periods of the life course are more important than others.

Methods We followed 3,951 men and 3,601 women born in Uppsala, Sweden, in 1915-1929 with known SEP at birth (age $0)$, during childhood (10 years), in adulthood (30-45 years) and in later life (50-65 years) from September 1980 until emigration, death, or until December 2010. Data on parents', partner's and own occupational status (a measure of SEP), marital status, deaths and emigrations were abstracted from birth records, parish records, school records, Census 1930 and routine registers. From the eligible sample who were alive and living in Sweden in September $1980 \quad(n=11,336), \quad 67 \%$ $(n=7552)$ had SEP recorded at all four-time points. We compared a set of nested Cox proportional regression models, each corresponding to a specific life course model (critical, sensitive and accumulation models), to a fully saturated model, to ascertain which model best describes the relationship between SEP and mortality. An alternative analysis employed latent class trajectories of SEP across same four time points. Analyses were stratified by gender.

Results The effect of SEP across the life course on all-cause mortality was best described by the sensitive period model in both genders with social advantage in later life (ages 50-65 years) having the largest protective effect (HR 0.80, 95\% CI $0.73-0.87$ in men and HR 0.82 , 95\% $0.75-0.91$ in women). A linear accumulation model also provided a good fit of the data for women. Only 5\% and 12\% of individuals experienced downward and upward social mobility during childhood respectively. The sensitive period model indicated that being advantaged at age 10 appeared to be more protective than at birth for males, while there was no difference between SEP at birth and age 10 in their effect on all-cause mortality among women. Additional adjustments for marital status did not affect the results appreciably and main results were also consistent with analyses that employed latent class trajectories of SEP.

Conclusion Our results lead to a conclusion that an individual's socio-economic position over the life course, including during early childhood does affect their risk of all-cause mortality in later life. These findings indicate that improvements in social conditions at any stage of the life course can contribute to reducing mortality at old age.

\section{OP41 THE HEALTH OF PEOPLE EXPERIENCING MULTIPLE FORMS OF SOCIAL EXCLUSION: A SYSTEMATIC REVIEW}

${ }^{1}$ EJ Tweed $*,{ }^{2} \mathrm{C}$ Sumpter, ${ }^{1} \mathrm{R}$ Thomson, ${ }^{3} \mathrm{D}$ Lewer, ${ }^{4} \mathrm{P}$ Southworth, ${ }^{5} \mathrm{~A}$ Kirolos, ${ }^{6} \mathrm{~A}$ Story, ${ }^{7} \mathrm{~A}$ Hayward, ${ }^{8} \mathrm{~S}$ Hwang, ${ }^{9} \mathrm{R}$ Aldridge, ${ }^{1} \mathrm{SV}$ Katikireddi. ${ }^{1} \mathrm{MRC} / \mathrm{CSO}$ Social and Public Health Sciences Unit, University of Glasgow, Glasgow, UK; ${ }^{2}$ Department of Public Health, NHS Forth Valley, Stirling, UK; ${ }^{3}$ Collaborative Centre for Inclusion Health, University College London, London, UK; ${ }^{4}$ NHS Dumfries and Galloway, UK; ${ }^{5}$ Usher Institute, University of Glasgow, Glasgow UK; ${ }^{6}$ University College London Collaborative Centre for Inclusion Health and UCLH Find and Treat Service London, UK; ${ }^{7}$ Institute of Epidemiology and Health Care, University College London, London, UK; ${ }^{8}$ Centre for Urban Health Solutions, St. Michael's Hospital and Department of Medicine, University of Toronto, Toronto, Canada; ${ }^{9}$ Institute of Health Informatics, University College London, London, UK

\subsection{6/jech-2019-SSMabstracts.43}

Background People with a history of homelessness, imprisonment, substance use, sex work, or serious mental illness experience much higher rates of ill-health and premature death than the general population. There is substantial overlap in these experiences in the population, and they may interact in important ways to influence health. However, the health outcomes associated with these experiences in combination have not previously been reviewed.

We therefore aimed to synthesise existing evidence on allcause mortality; cause-specific mortality; morbidity from conditions appearing in ICD-10; self-rated health; and quality of life among people with lifetime exposure to more than one of the following: homelessness; imprisonment; substance use; sex work; or serious mental illness.

Methods We searched Medline, Embase, and Psycinfo using search terms for the above exposures and outcomes, in consultation with a medical librarian. Eligible studies comprised peer-reviewed English-language articles from high-income countries published since 1998 reporting at least one relevant outcome for people with lifetime exposure to two or more exposures of interest, in comparison to people with one or no exposures. Screening was undertaken independently by two authors using Covidence, with risk of bias assessed using a modified Newcastle-Ottawa Scale. Findings were summarised using a pre-specified narrative synthesis plan. The protocol was registered with PROSPERO (CRD42018097189).

Results Searches retrieved 15,948 unique citations. After full text screening of 1,583 studies, initial results from 293 studies for which data extraction has been completed are presented here. Of these, $73 \%$ were cross-sectional studies. The most common exposure combinations were imprisonment \& substance use (33\%) and serious mental illness \& substance use (26\%); only 11 data points (1\%) reported outcomes associated 